\title{
Suscetibilidade de Rhyzopertha dominica (Fabricius) (Coleoptera: Bostrichidae) ao enxofre
}

\author{
Susceptibility of Rhyzopertha dominica (Fabricius) (Coleoptera: Bostrichidae) to sulfur
}

\author{
José Roberto Gonçalves ${ }^{\mathrm{I}}$ Lêda Rita D’Antonino Faroni ${ }^{\mathrm{II}}$ Raul Narciso Carvalho Guedes ${ }^{\mathrm{I}}$ \\ Carlos Romero Ferreira de Oliveira ${ }^{\mathrm{I}}$ Ramon Macedo Silva ${ }^{\mathrm{II}}$
} \begin{abstract}
RESUMO
As criações de Rhyzopertha dominica (Fabricius) (Coleoptera: Bostrichidae) são freqüentemente infestadas pelo ácaro Acarophenax lacunatus (Cross e Krantz) (Prostigmata: Acarophenacidae). O objetivo deste trabalho foi avaliar doses de enxofre, acaricida eficaz contra A. lacunatus, nãoprejudiciais ao desenvolvimento de $\boldsymbol{R}$. dominica. As unidades experimentais foram constituídas de placas de Petri contendo $30 \mathrm{~g}$ de grãos de trigo infestados com 30 adultos de $\boldsymbol{R}$. dominica. Os tratamentos consistiram na utilização de doses de enxofre sobre os grãos, correspondentes a 0,$0 ; 0,6 ; 0,9 ; 1,2 ; 1,5 ; 3,0$; 6,$0 ; 12,0 ; 24,0$ e 48,0mg i a $\mathrm{g}^{-1}$, em dez repetições. As unidades experimentais foram armazenadas por 60 dias a $30 \pm 1^{\circ} \mathrm{C}$, $60 \pm 5 \%$ UR e escotofase de 24h. O desenvolvimento de $\boldsymbol{R}$. dominica foi afetado pela utilização de doses de enxofre maiores que 3,0mg i a $\mathrm{g}^{-1}$.
\end{abstract}

Palavras-chave: Insecta, criação massal, Acarophenacidae, acaricida.

\section{ABSTRACT}

The laboratory rearing of Rhyzopertha dominica (Fabricius) (Coleoptera: Bostrichidae) is frequently infested by the parasite mite Acarophenax lacunatus (Cross and Krantz) (Prostigmata: Acarophenacidae). This study was aimed at evaluating the sulfur doses, an effective acaricide against $\boldsymbol{A}$. lacunatus, not harmful to the development of with $\boldsymbol{R}$. dominica. The experimental units were Petri dishes containing $30 \mathrm{~g}$ of whole wheat grains powdered with the different doses of the sulfur $(0.0,0.6,0.9,1.2,1.5,3.0,6.0,12.0,24.0$ and $48.0 \mathrm{mg}$ a $\mathrm{i}^{-1}$ ) infested with 30 adults of $\boldsymbol{R}$. dominica, in ten replicates. All treatments were maintained under controlled conditions $\left(30 \pm 1^{\circ} \mathrm{C}, 60 \pm 5 \% r h\right.$ and $24 \mathrm{~h}$ scotophase) for 60 days after the insect infestation. Sulfur doses higher than $3.0 \mathrm{mg} \mathrm{a} \mathrm{i} \mathrm{g}^{-1}$ negatively affected $\boldsymbol{R}$. dominica development.
Key words: Insecta, rearing mass, Acarophenacidae, acaricide.

A criação de insetos-praga em condições de laboratório é uma prática corriqueira e de suma importância para seu estudo (PARRA, 2002), estando sujeita a contaminações por ácaros, microrganismos ou até mesmo por outras espécies de insetos que não se pretende multiplicar (ARNAUD et al., 1996). Estas complicações se agravam ainda mais à medida que se aumenta o número de insetos produzidos. Com isto, o monitoramento constante das criações se faz necessário para que seja possível direcionar uma decisão por parte do laboratorista, a fim de garantir a qualidade e/ou proteção dos organismos criados (HANKS et al., 1992).

A limpeza das salas de multiplicação de insetos e o monitoramento das condições climáticas destes ambientes são importantes para a prevenção de contaminações nas criações (FLECHTMANN \& ZEM, 2002). Existem situações em que se torna necessária a utilização de pesticidas para proteger criações de insetos contra o ataque de ácaros parasitas, uma vez que estes organismos se multiplicam em grande número sobre essas criações, podendo causar sérios prejuízos e até a sua perda (NAVARRO et al., 1983).

O uso de agrotóxicos sobre criações de insetos requer estudos prévios para avaliar a compatibilidade destes produtos com os organismos a serem multiplicados. NAVARRO et al. (1983) observaram

\footnotetext{
IDepartamento de Biologia Animal, Universidade Federal de Viçosa (UFV), 36570-000, Viçosa, MG, Brasil.

${ }^{I I}$ Departamento de Engenharia Agrícola, Universidade Federal de Viçosa (UFV), 36570-000, Viçosa, MG, Brasil. E-mail: goncalves_mip@hotmail.com. Autor para correspondência.
} 
que o uso do acaricida Galecron 50 CE (chlordimeform) para proteger criações de Sitotroga cerealella (Olivier) (Lepidoptera: Gelechiidae) contra o ácaro parasita Pyemotes spp. (Acari: Pyemotidae) não permitiu que larvas desse lepidóptero penetrassem nos grãos de trigo. Esses autores também verificaram que o uso do produto Kelthane 18,5 CE (dicofol) não apresentou diferença em relação à testemunha, não tendo afetado a população de insetos adultos. Resultados semelhantes foram observados por HANKS et al. (1993), utilizando enxofre sobre colônias de Phoracantha semipunctata (Fabricius) (Coleoptera: Cerambycidae), mantendo-as protegidas da infestação de Pyemotes spp., que afeta seriamente a criação desse coleóptero.

Muitas espécies de insetos são utilizadas para multiplicação de inimigos naturais, e o coleóptero Rhyzopertha dominica (Fabricius) (Coleoptera: Bostrichidae) vem sendo usado na manutenção de populações do ácaro parasita Acarophenax lacunatus (Cross e Krantz) (Prostigmata: Acarophenacidae). No entanto, quando se pretende fazer criações de $\boldsymbol{R}$. dominica livres desse ácaro, surge uma série de dificuldades devido à elevada capacidade de infestação de $\boldsymbol{A}$. lacunatus (GONÇALVES et al., 2002).

Estudos preliminares de laboratório demonstraram que doses de enxofre acima de $0,3 \mathrm{mg}$ i a $\mathrm{g}^{-1}$ são eficazes no controle de A. lacunatus (GONÇALVES, 2005). Ao se selecionar um acaricida para utilização sobre criações de insetos, deve-se lembrar que esta substância não pode afetar o organismo que se deseja multiplicar. Assim, o objetivo deste estudo foi avaliar o efeito de diferentes doses de enxofre sobre o desenvolvimento de $\boldsymbol{R}$. dominica.

Exposição de R. dominica ao Enxofre. As unidades experimentais consistiram de placas de Petri de 140 x 10mm (diâmetro x altura), contendo 30g de grãos de trigo com teor de água de $13 \%$ b.u. (base úmida), infestados com 30 adultos não-sexados de $\boldsymbol{R}$. dominica, com idades entre três e sete dias. Os tratamentos consistiram na utilização de doses de enxofre em pó (grau de pureza 99\%) sobre grãos de trigo, correspondentes a 0,$0 ; 0,6 ; 0,9 ; 1,2 ; 1,5 ; 3,0 ; 6,0$; 12,0; 24,0 e 48,0mg do ingrediente ativo (i a) $\mathrm{g}^{-1}$, em dez repetições. As placas foram revestidas com filme plástico de PVC para evitar a fuga dos insetos. Todos os tratamentos foram armazenados por 60 dias, em câmara climática ajustada $\left(30 \pm 1^{\circ} \mathrm{C}, 60 \pm 5 \%\right.$ UR e escotofase de 24h).

Após o período de armazenamento, as populações de $\boldsymbol{R}$. dominica e a massa de grãos de trigo foram avaliadas. Para isso, a massa de grãos de cada placa foi passada em peneira de orifícios de $1 \mathrm{~mm}$ de diâmetro, separando-se os insetos dos grãos e o resíduo (pó) contendo fases imaturas (ovos e larvas) de $\boldsymbol{R}$. dominica. Em seguida, procedeu-se à determinação da perda de massa dos grãos de trigo (\%) através da alteração da massa com o término do experimento e a contagem do número de adultos vivos de $\boldsymbol{R}$. dominica. Para quantificar o número de ovos e larvas de primeiro ínstar de $\boldsymbol{R}$. dominica, foi analisado o resíduo da massa de grãos com o auxílio de estereoscópio.

A taxa instantânea de crescimento de $\boldsymbol{R}$. dominica $\left(\mathrm{r}_{\mathrm{i}}\right)$ foi calculada usando a equação:

$$
r_{i}=\left\lfloor\ln \left(N_{f} / N_{0}\right)\right\rfloor \Delta t
$$

onde $\mathrm{N}_{\mathrm{f}}$ = número final de insetos vivos; $\mathrm{N}_{0}=$ número inicial de insetos vivos; $\ddot{A}$ = variação de tempo (número de dias em que o ensaio foi executado) (WALTHALL \& STARK, 1997).

Para avaliar o efeito das doses de enxofre sobre o desenvolvimento de $\boldsymbol{R}$. dominica, foi realizada análise de regressão. A perda de massa dos grãos de trigo e a taxa instantânea de crescimento (r) de $\boldsymbol{R}$. dominica, assim como o número de ovos e larvas deste coleóptero, foram comparados por análise de correlação de Pearson.

A $r_{i}$ de $\boldsymbol{R}$. dominica apresentou diferença significativa para as doses de enxofre utilizadas $\left(\mathrm{F}_{11 ; 108}\right.$ $=20,37 ; \mathrm{P}<0,001$ ) e correlação positiva para a perda de massa dos grãos de trigo $(\mathrm{r}=0,84 ; \mathrm{P}=0,0001)$. Na dose de enxofre igual ou maior que $3,0 \mathrm{mg}^{\mathrm{i}} \mathrm{a} \mathrm{g}^{-1}$, houve tendência decrescente mais acentuada da $r_{i}$ deste coleóptero; no entanto, entre as doses de 0,6 a 3,0mg i

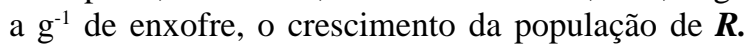
dominica não foi afetado (Figura 1).

No que se refere ao número de ovos de $\boldsymbol{R}$. dominica, também foi observada diferença significativa com a utilização do enxofre $\left(\mathrm{F}_{11 ; 108}=67,50 ; \mathrm{P}<0,001\right) \mathrm{e}$ correlação positiva com o número de larvas de primeiro ínstar deste inseto $(\mathrm{r}=0,51 ; \mathrm{P}=0,0001)$. As doses de enxofre menores que $6,0 \mathrm{mg}^{\mathrm{i}}$ a $\mathrm{g}^{-1}$ não afetaram as proporções de imaturos de $\boldsymbol{R}$. dominica (Figura 2).

Neste trabalho, buscaram-se, por meio da aplicação de enxofre em grãos de trigo, doses que não prejudicassem o desenvolvimento de $\boldsymbol{R}$. dominica. Nota-se, de maneira geral, que o uso de doses abaixo de 3,0mg i a g ${ }^{-1}$ não afetou as criações deste coleóptero. Isso é importante, uma vez que já foi constatada a suscetibilidade de A. lacunatus a doses de enxofre acima de 0,3mg i a g-1 (GONÇALVES, 2005), podendose obter criações de $\boldsymbol{R}$. dominica livres da infestação deste ácaro. Resultados semelhantes foram observados para $\boldsymbol{P}$. semipunctata e $\boldsymbol{S}$. cerealella, que foram protegidas da infestação de Pyemotes spp. por meio da utilização de enxofre e dicofol, respectivamente (NAVARRO et al., 1983; HANKS et al., 1993). 


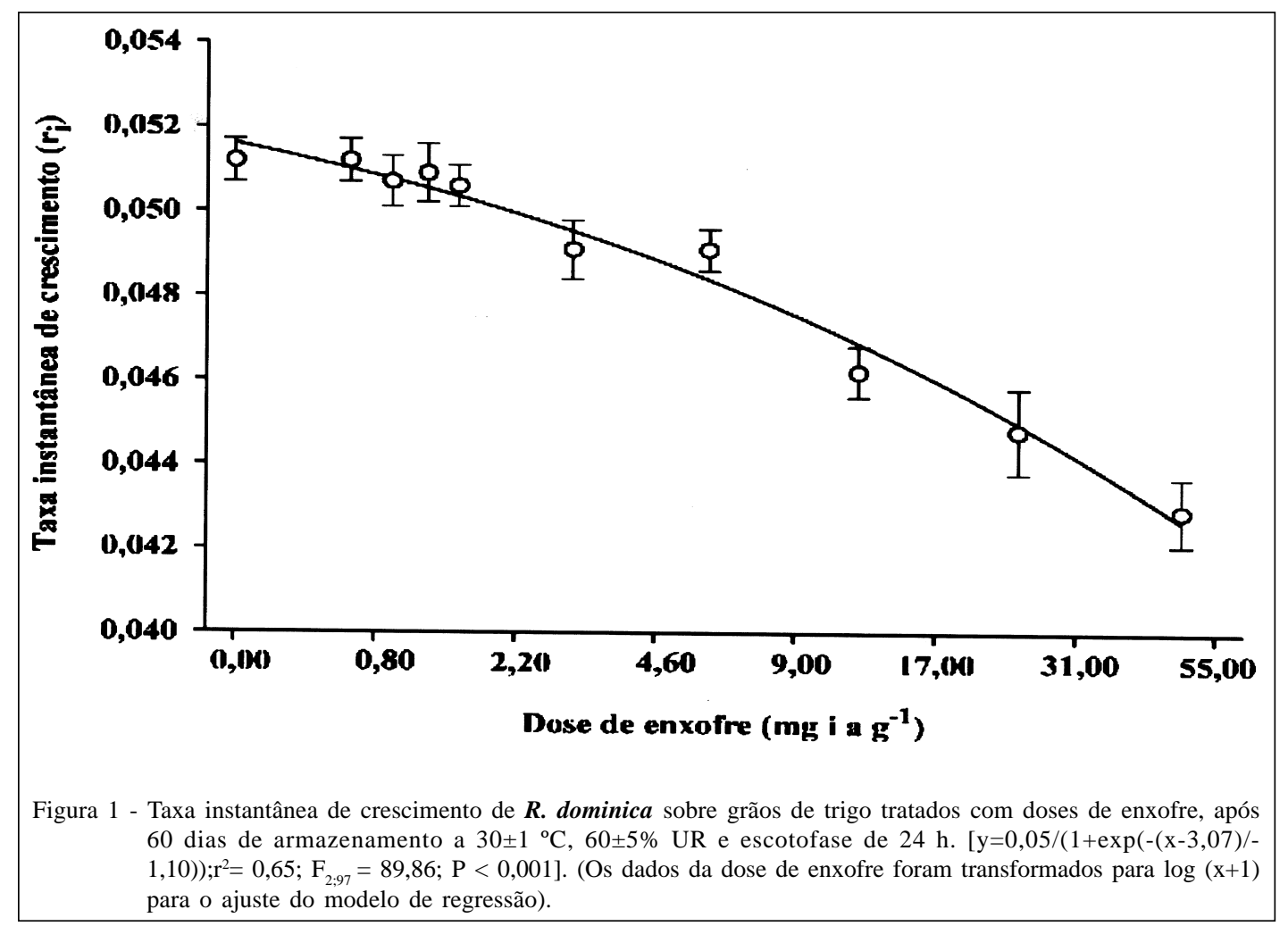

As criações massais de insetos em laboratório estão sujeitas a infestações por diversos tipos de pragas, ou seja, outras espécies de insetos que não se pretende multiplicar, ácaros e microrganismos (ARNAUD et al., 1996). Assim, devem ser tomadas medidas de higiene para todos os equipamentos utilizados na multiplicação dos insetos, assepsia na preparação de dietas artificiais ou, quando a dieta for natural (grãos), deve-se ter cuidado com o seu teor de água. Além disso, antes de se iniciar uma criação de insetos, os grãos devem passar por um tratamento curativo (fumigação), e a temperatura e a umidade relativa do ambiente devem ser monitoradas de modo que sejam desfavoráveis ao desenvolvimento de ácaros e microrganismos (FLECHTMANN \& ZEM, 2002). Mesmo tomando todos estes cuidados, $\boldsymbol{A}$. lacunatus infesta as criações de $\boldsymbol{R}$. dominica devido ao fato de este ácaro apresentar exigências climáticas semelhantes às de seu hospedeiro. Somando-se a isso, os ácaros da família de $\boldsymbol{A}$. lacunatus apresentam elevada capacidade de dispersão devido ao processo de foresia, podendo, assim, deslocar-se por longas distâncias (STEINKRAUS \& CROSS, 1993). Dessa forma, tornase necessário o uso de um acaricida que seja eficaz contra A. Iacunatus e não influencie no desenvolvimento de $\boldsymbol{R}$. dominica.

O uso de agrotóxicos sobre criações de insetos é uma ferramenta indispensável em determinadas situações. Uma das dificuldades para este manejo é a inexistência de produtos registrados para este fim. Embora existam alguns produtos recomendados, poucos estudos retratam seus efeitos sobre as criações de insetos (NAVARRO et al., 1983).

O uso do enxofre, apesar de apresentar pouco efeito sobre os insetos, demonstra eficácia sobre ácaros (HANKS et al., 1992). Entretanto, como visto neste estudo, doses elevadas desse acaricida podem causar reduções no número de adultos e imaturos de $\boldsymbol{R}$. dominica e, conseqüentemente, menor incremento populacional desses organismos. Estes resultados foram semelhantes aos observados por outros pesquisadores, que constataram efeito deletério do enxofre sobre Trichogramma spp. (Hymenoptera: Trichogrammatidae) (THOMSON et al., 2000) e Podisus nigrispinus (Hemiptera: Pentatomidae) (Dallas) (TORRES et al., 2002). Portanto, embora o enxofre seja um acaricida importante para ser utilizado em criações de insetos, deve-se ter cuidado com a dose deste produto, uma vez que pode causar prejuízos para os insetos que se deseja criar.

O monitoramento da evolução de pragas em criações de insetos é de fundamental importância, pois permite detectar o início da infestação e direcionar a tomada de decisão por parte do pesquisador, a fim de garantir a qualidade e a proteção dos organismos criados. Neste estudo, encontrou-se uma alternativa simples e barata para prevenir e/ou controlar a infestação do ácaro A. lacunatus, sem afetar o desenvolvimento de $\boldsymbol{R}$. dominica.

Ciência Rural, v.37, n.4, jul-ago, 2007. 




O uso de doses de enxofre menores que $3,0 \mathrm{mg}$ i a $\mathrm{g}^{-1}$ não afetam negativamente o desenvolvimento de $\boldsymbol{R}$. dominica. Com isso, devemse usar doses de enxofre entre 0,3 e $6,0 \mathrm{mg} \mathrm{i} \mathrm{a} \mathrm{g}^{-1}$ para o controle do ácaro parasita sem afetar-se significativamente seu hospedeiro.

\section{REFERÊNCIAS}

ARNAUD, L. et al. A simple technique to relieve Tribolium castaneum (Coleoptera: Tenebrionidae) of Acarophenax tribolii (Acarina: Pyemotidae). Tribolium Information Bulletin, San Bernardino, v.36, p.86-87, 1996.

FLECHTMANN, C.H.W.; ZEM, A.C. Ácaros de produtos armazenados. In: LORINI, I. et al. Armazenagem de grãos. Campinas: Instituto Bio Geneziz, 2002. Cap.10, p.807-856.

GONÇALVES, J.R. Interações de Acarophenax lacunatus com deltametrina, temperatura e o parasitóide Anisopteromalus calandrae na supressão populacional de Rhyzopertha dominica. 2005. 91f. Tese (Doutorado em Entomologia) Curso de Pós-graduação em Entomologia, Universidade Federal de Viçosa.

GONÇALVES, J.R. et al. Pyrethroid-Acarophenax lacunatus interaction in suppressing the beetle Rhyzopertha dominica on stored wheat. Experimental \& Applied Acarology, Netherlands, v.26, n.1, p.231-242, 2002.

HANKS, L.M. et al. Control of the straw itch mite (Acari: Pyemotidae) with sulfur in an insect rearing facility. Journal of Economic Entomology, Lanham, v.85, n.3, p.683-686, 1992.
HANKS, L.M. et al. Phoracantha semipunctata (Coleoptera: Cerambycidae), a serious pest of Eucalyptus in California: Biology and laboratory rearing procedures. Annals of the Entomological Society of America, Lanham, v.86, n.1, p.96102, 1993.

NAVARRO, R. et al. Prueba de diferentes substratos para la cria masiva de Sitotroga cerealella. Agronomía Tropical, Maracay, v.33, n.1, p.111-121, 1983.

PARRA, J.R.P. Criação massal de inimigos naturais. In: PARRA, J.R.P. Controle biológico no Brasil: parasitóides e predadores. São Paulo: Manole, 2002. Cap.9, p.143-164.

STEINKRAUS, D.C.; CROSS. E.A. Description and life history of Acarophenax mahunkai, n. sp. (Acari, Prostigmata: Acarophenacidae), an egg parasite of the lesser mealworm (Coleoptera: Tenebrionidae). Annals of the Entomological Society of America, Lanham, v.86, n.1, p.239-249, 1993.

THOMSON, L.J. et al. Effects of sulfur on Trichogramma egg parasitoids in vineyards: measuring toxic effects and establishing release windows. Australian Journal of Experimental Agriculture, Collingwood, v.40, n.8, p.1165-1171, 2000.

TORRES, J.B. et al. Compatibilidade de inseticidas e acaricidas com o percevejo predador Podisus nigrispinus (Dallas) (Heteroptera: Pentatomidae) em algodoeiro. Neotropical Entomology, Londrina, v.31, n.2, p.311-317, 2002.

WALTHALL, W.K.; STARK. J.D. Comparison of two population level ecotoxicological endpoints: The intrinsic $\left(r_{m}\right)$ and instantaneous $\left(r_{i}\right)$ rates of increase. Environmental Toxicology and Chemistry, Pensacola, v.16, n.5, p.10681073, 1997.

Ciência Rural, v.37, n.4, jul-ago, 2007. 